\title{
Critical Current Degradation in Superconducting Materials with Nano-Defects Induced by Heavy Ions Irradiation
}

\author{
Sosnowski J \\ Electrotechnical Institute, Pożaryskiego 28, 04-703 Warsaw, Poland
}

Received: March 06, 2014; Accepted: July 11, 2014; Published: July 16, 2014

*Corresponding author: Sosnowski J, Electrotechnical Institute, Pożaryskiego 28, 04-703 Warsaw, Poland, E-mail: sosnow@iel.waw.pl

\begin{abstract}
This paper has been considered an influence of the nano-defects created in the process of the heavy ions or fast neutrons irradiation on the current-voltage characteristics and critical current of multilayered high temperature superconducting materials. Theoretical model based on an energy balance analysis for the system of the vortex captured on pinning centre has been presented and used for the critical current description. The regular arrangement of the columnar type defects caused by irradiation has been considered. In the model has been taken into account, a Lorentz force acting on pancake type vortices induced by current flow and elasticity energy of deformed vortex lattice. The calculations of the I-V characteristics and critical current have been performed in the function of nano-sized defects dimensions and their concentration. The results of calculations indicate in which way the degradation of critical current occurs under the irradiation process for HTc sample with optimal concentration of internal defects. For such optimally prepared tapes the irradiation leads to the decrease of the critical current and appropriate change of the current-voltage characteristics. Elaborated model has been applied to the second generation tapes. While it is possible extend it also on tapes of the first generation and low temperature wires.
\end{abstract}

Keywords: Nano-Sized defects; HTc Superconductivity; Neutrons irradiation; Critical current

\section{Introduction}

Physical properties of the superconducting materials, both high temperature HTc as well as low temperature LTc superconductors are very sensitive to their stoichiometry including existence of structural defects. While chemical composition is responsible mainly for critical temperature of superconductor, second crucial from applied point of view parameter of superconductors, which is critical current is very strongly dependent on the nano-sized defects appearance. The nano-defects in superconducting tapes can arise in the nuclear physics devices, such as accelerators, with the superconducting windings focusing the ionic beam and in current leads. In these devices superconducting materials are frequently exposed to the direct irradiation by the heavy ions or fast neutrons, creating defects of the nanometric size, it is nano-defects. But it is not unique way of creation the nano-sized defects in superconductors. They arise too in the usual technological process of fabrication these wires and their handling, as is shown in Figure 1. Black points indicate mainly nano-pores and $\mathrm{CuO}_{2}$ grains. During the winding process of the superconducting coils the defects in the superconductors will arise in the form of the micro crushes. These nano-defects appearing in the superconducting tapes used in the windings of the superconducting magnets of the accelerators influence their proper work through the pinning interaction, enhancing the critical current but too large ions bombardment damages the structure of superconductor. It is important task therefore to investigate function and mechanism of the degradation of the critical current under the irradiation existence. It concerns especially HTc superconducting tapes characterised by $2 \mathrm{D}$ superconductivity mechanism, as well as $\mathrm{Nb}_{3} \mathrm{Sn}$, which are also sensitive to irradiation creating disorder in linear chains of $\mathrm{Nb}$ atoms, responsible for superconductivity. For NbTi materials indicating 3D mechanism of superconductivity irradiation effects should be not so important from the superconducting critical temperature point of view. Onto relevance of this pinning interaction for properties of superconductors indicate many papers devoted to this field, from which are referred here [1-7].

\section{Model of the Current-Voltage Characteristics}

Theoretically influence of heavy ions irradiation on the

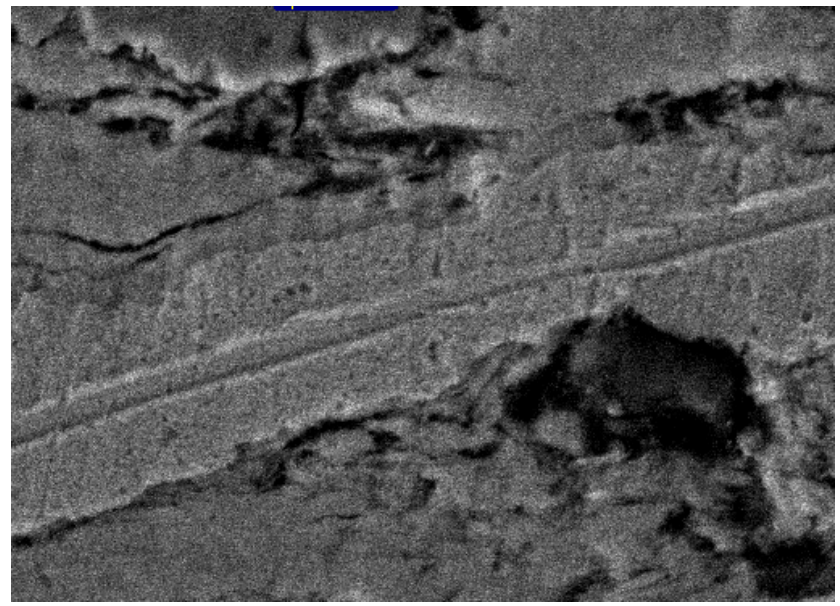

Figure 1: Scanning Electron Microscopy (SEM) image of the cross-section, superconducting Bi-based tape, indicating an existence of nanostructural defects. The length of the cross-section is about $50 \mu \mathrm{m}$. 
critical current of multi-layered HTc superconductors, in which structure pancake type vortices are generated, has been considered basing on an analysis of the energy balance of the system of captured vortex in the nano-defect, caused by the heavy ions irradiation. Described geometry is presented in Figure 2. Cylindrical defects shown here schematically are generated by irradiation of superconducting material in the form of tape or film by heavy ions, while rectangular defects can arise during the winding procedure of the superconducting coils, leading to the micro-crashes generation, as it was stated previously. Shift of the vortex from the captured, equilibrium position, as shown in Figure 2 causes an increase of the normal energy of the system [8-11]. From the other side pinning of an individual vortex leads to its displacement in the regular vortex lattice and causes an enhancement in the elasticity energy of the system because the regular vortex lattice is disturbed then. Also current flow leads to rise of the Lorentz force tearing the pancake vortices, which start to diffuse inside the superconductor [12]. Energy barrier appears then, in which each pancake shape vortex should pass in the flux creep process. An enhancement of the energy of system connected with the normal state development during the shift of the pancake type vortex, leads to potential well appearance and is given for defects of cylindrical geometry by an expression:

$$
U=\frac{\mu_{0} H_{c}^{2} l}{2}\left[\xi^{2}\left(\alpha-\pi-\frac{\sin 2 \alpha}{2}\right)-R_{0}^{2}\left(\beta-\frac{\sin 2 \beta}{2}\right)\right]
$$

In Equation 1 has been used notation shown in Figure 2. In the case of large diameters of defects and especially microcrushes flat geometry of the pinning centres shown in left part of Figure 2 can be considered. For that geometry potential well shape is given by:

$$
U=\frac{\mu_{0} H_{c}^{2} l \xi^{2}}{2}\left(\alpha-\pi-\frac{\sin 2 \alpha}{2}\right)
$$

In Equations 1-2 has been applied energy scaling in such a way that normal state energy is on zero level. Then the depth of the pinning potential well is equal to the condensation energy because it is just the gain of the system energy with totally pinned vortex. $H_{c}$ denotes thermodynamic magnetic field, angles $\alpha$ and $\beta$ shown in Figure 2, describe the deflection of the vortex core against its equilibrium position in the pinning centre. $R_{0}$ is the radius of the pinning centre - columnar defect;
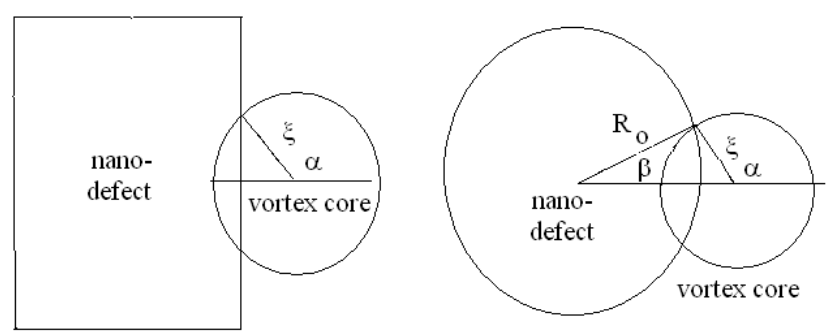

Figure 2: Scheme of the geometry of the interaction of the pancake vortex of the core radius equal to the coherence length $\xi$ captured on the rectangular (left) and cylindrical (right) defect. coherence length $\xi$ describes the radius of the core of the vortex, while $l$ is the thickness of the vortex, for HTc superconductors of the thin pancake form, approximated by the thickness of the superconducting layer. The pinning potential determined above is supplemented by the Lorentz force potential, connected with the interaction of the local current density surrounding vortex and magnetic induction $B$, tearing it off from an initial, equilibrium capture position. It has been considered here an initial vortex position onto the depth of the coherence length $\xi$ inside the pinning centre, which is favorable from the point of view of the shielding currents configuration. Potential well is also influenced by vortex lattice elasticity energy, as it was stated previously. The tilting of the potential energy wells, given by Equation 2 caused by the current flow and Lorentz force potential leads to the expression for an energy barrier, which should cross vortex in the flux creep process, in the function already of the reduced current density $\boldsymbol{i}=\boldsymbol{j} / \boldsymbol{j}_{\boldsymbol{c}^{\prime}} \boldsymbol{j}$ is here transport current density, while $\boldsymbol{j}_{\boldsymbol{c}}$ critical current density expressing the transitions between flux creep and flux flow states:

$$
j_{c}=\frac{\mu_{0} H_{c}^{2}}{\pi \xi B} \cdot \frac{S\left(1-S / d^{2}\right)}{d^{2}}
$$

$\boldsymbol{B}$ is applied magnetic induction, $\boldsymbol{\mu}_{\boldsymbol{0}}$ magnetic permeability. Parameter $\boldsymbol{S}$ is the cross-section of nano-defect, while $\boldsymbol{d}$ the lattice constant of the regularly arranged nano-defects into the square lattice. Potential barrier height is given then as follows for flat defects:

$$
\Delta U=\frac{\mu_{0} H_{c}^{2}}{2} l \xi^{2}\left[-\arcsin \left(\frac{j}{j_{c}}\right)+\frac{\pi}{2}-\frac{j}{j_{c}} \sqrt{1-\left(\frac{j}{j_{c}}\right)^{2}}\right]
$$

Potential barrier $\Delta \mathrm{U}$ given by Equation 4 is strongly dependent on the kind of defects, their size and concentration and determines the shape of the current-voltage characteristics of the superconductors:

$$
E=-B \omega d\left[\exp \left[-\frac{\Delta U(0)}{k_{B} T}\left(1+\frac{j}{j_{C}}\right)\right]-\exp \left(-\frac{\Delta U}{k_{B} T}\right)\right]
$$

$E$ is electric field, while $\omega$ the flux creep frequency, $k_{B}$ Boltzmann's constant, $T$ temperature.

Equation 5 describes both forward as well as backward flux creep processes and has been used according to presented model for investigations of the current-voltage characteristics and then critical current, filling electric field criterion, dependence on the heavy ions irradiation. This task has great importance for an improvement of our knowledge on the work of the superconducting windings in accelerators used in nuclear physics investigations. As the result of irradiation the columnar defects are created in superconducting tapes. In model it was assumed that these defects, acting as pinning centres form square array. Their lattice constant is inversely proportional then to the square root from the surface concentration of the defects. The results of the numerical calculations of the current-voltage characteristics of the HTc superconducting tape under influence of irradiation 
are shown in Figures 3-4. Figure 3 presents the influence of the irradiation dose on the current-voltage characteristics of HTc superconductor with optimal initial concentration of inherent defects. Current-voltage characteristics are strongly shifted then, which indicates that the tape is passing onto the over-doped with nanodefects region, while its structure is damaged.

In Figure 4 from other side is shown the influence of the diameter of the nano-sized defects on the current-voltage characteristics of HTc superconductor. Also in this case strong influence of this parameter is observed here.

At an aim of comparison the theoretical results predicted by proposed model with experimental data the calculations have been peformed of the influence of the static magnetic field amplitude on the current-voltage characteristics. Measured I-V characteristics on $\mathrm{YBaCuO}$ sample in static magnetic field, of value given at each curve are shown in Figure 5. Temperature of measurements was $77 \mathrm{~K}$, while critical temperature of superconducting ceramic above $90 \mathrm{~K}$. These measurements are in qualitative accordance with theoretical predictions of model presented in the Figure 6

\section{Influence of Created by Heavy Ions Irradiation Defects on the Critical Current}

Above described theoretical model has been used then for determining the influence of the irradiation by heavy ions and fast neutrons on the critical current of HTc tapes. The irradiation changes the lattice constant of the defects array influencing the critical current density given by Equation 3 as well as critical current filling electric field criterion, described by Equation 5 and diminishes therefore the superconductor volume. Calculations of the critical current as well as currentvoltage characteristics have been performed for optimally initially defected sample, in the technological process, as shown in Figure 1. Then additional concentration of nano-defects arising through the ions bombarding process destroys mainly superconductivity. Simplified model leading to the expression 3 for critical current, based on individual interaction of vortex with pinning centre has been extended on the case of the regular ordering of defects into lattice, taking into account the decrease

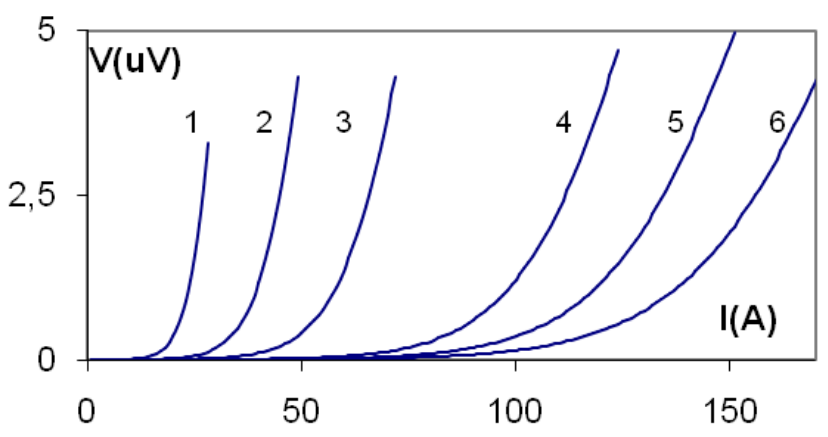

Figure 3: Influence of the nano-sized defects on the current-voltage characteristic of HTc superconductor optimally initially doped with nano-defects, for surface concentration of defects equal to: (1) $105 \cdot 10^{10}$ $\mathrm{cm}^{-2}$, (2) $99 \cdot 10^{10} \mathrm{~cm}^{-2}$, (3) $92,5 \cdot 10^{10} \mathrm{~cm}^{-2}$, (4) $80 \cdot 10^{10} \mathrm{~cm}^{-2}$, (5) $74 \cdot 10^{10}$ $\mathrm{cm}^{-2},(6) 68 \cdot 10^{10} \mathrm{~cm}^{-2}$.

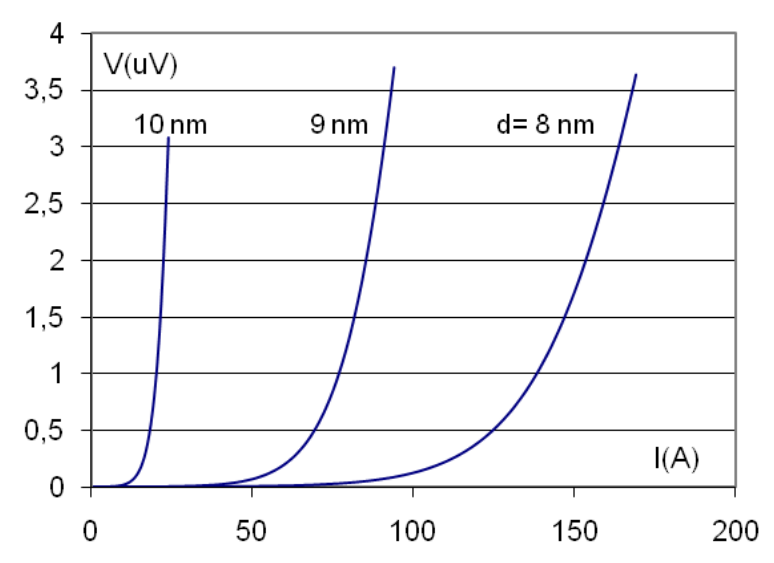

Figure 4: The influence of the dimension of the nano-sized defects on the current-voltage characteristics of HTc superconductor

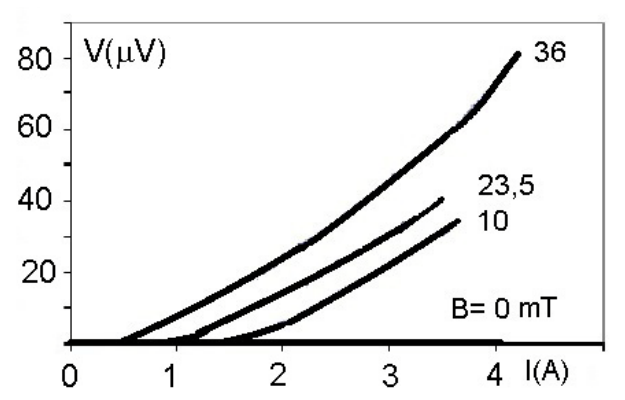

Figure 5: Measured in liquid nitrogen temperature magnetic field dependence of the current-voltage characteristics of $\mathrm{YBaCuO}$ superconducting ceramic. The static magnetic field values are given in milliteslas at each curve.

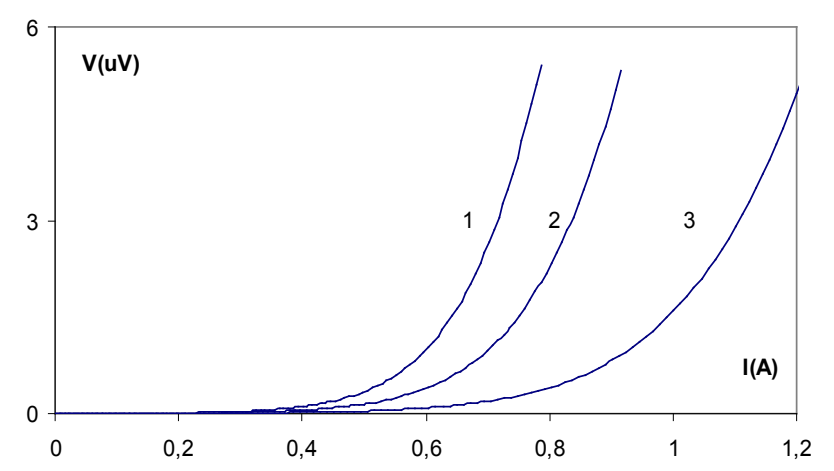

Figure 6: Calculated current-voltage characteristics of the HTc superconductor in static magnetic field of the amplitude: (1) B=21,5 mT, (2) 16,3 mT, (3) $8,5 \mathrm{mT}$.

of the cross-section of the deformed by nano-defects regions and increase of the pinning interaction. Then HTc superconducting tape of the second generation has been treated as long thin film composed from the regions of smaller cross-section, in which appear defects and regions non-deformed. The same current $I$ flow through both of these connected in raw domains, but different is already the current density $j$. Because these domains are electrically connected, so total electric field registered on the 
voltage taps is superposition of electric fields generated on each of them. For simplifying the calculations, especially concerning the separation of these two parts it has been assumed the square cross-section shape of the defects. The strong influence on the critical current of the division of tape onto un-defected and defected regions is presented in Figure 7. It is shown here the comparison of the dependence of the critical current received in the simple homogeneous approximation given by Equation 3 with modified inhomogeneous approach taking into account just existence of these two electrically connected in raw regions. For both cases it is observed strong decrease of the critical current with irradiation for optimally initially defected tape. This approximation concerns especially HTc superconducting tapes of the second generation $(2 \mathrm{G})$, in which superconducting current flows mainly in thin superconducting layer, strongly sensitive therefore to the nano-defects. On the other hand in the case of the first generation wires, thin filaments are immersed into the silver matrix. Then beside this partition effect should be additionally treated parallel connection of both un-deformed and deformed regions with the silver matrix, as is in the sharing current process. This should additionally modify the critical current dependence on irradiation. In Figure 8 is shown the critical current dependence not only on irradiation of heavy ions concentration but also on the nano-defects dimension, which is other important parameter.

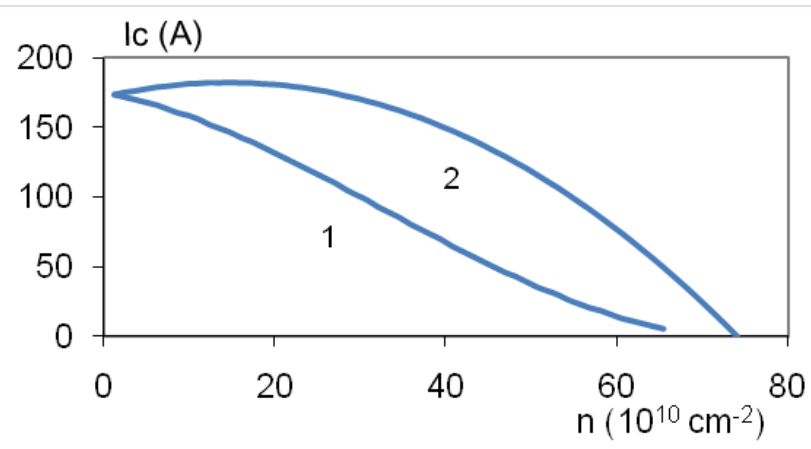

Figure 7: Theoretically predicted influence of the heavy ions irradiation on the critical current of the HTc superconducting tape for the model: (1) connected in raw un-defected and defected domains, (2) homogeneous approach.

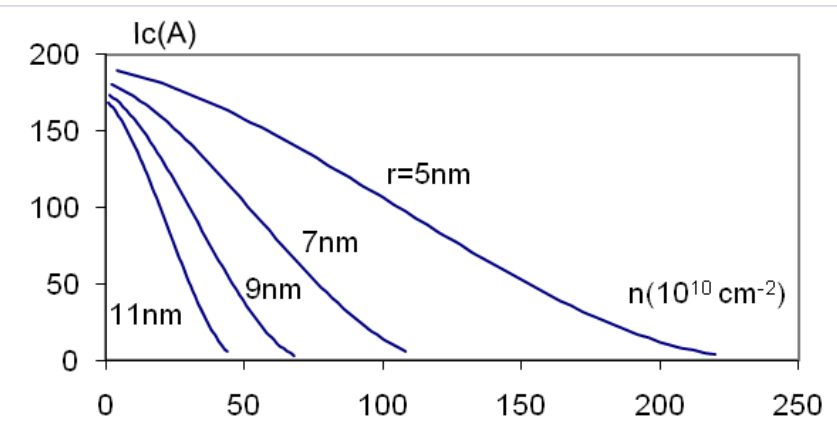

Figure 8: Calculated influence of the irradiation dose, creating nanosized defects of the dimensions given at each curve, on the critical current of HTc superconducting tape.

\section{Conclusions}

Summing up we state that results of present investigations indicate on the relevant meaning of heavy ions irradiation for the proper work of superconducting windings used in cryogenic accelerators. The case of HTc superconducting tapes of second generation was investigated, while this model can be applied too for the first generation tapes, as well as low temperature superconductors, especially $\mathrm{Nb}_{3} \mathrm{Sn}$ wires with linear chains of transitions atoms responsible for superconductivity.

\section{References}

1. Kim YB, Hempstead CF, Strnad AR. Phys Rev Letters. 1963; 131:2486.

2. Bean CP. Magnetization of Hard Superconductors. Phys Rev Letters. 1962; 8:250.

3. Okamura K, Kiuchi M, Otabe ES, Yasuda T, Matsushita T, Okayasu $\mathrm{S}$. The pinning property of Bi-2212 single crystals with columnar defects. Supercond Sci Technol, 2004; 17(1):S20. doi:10.1088/09532048/17/2/055.

4. Yamada H, Yamasaki H, Develos-Bagarinao K, Nakagawa Y, Mawatari $\mathrm{Y}$, Nie JC, et al. Flux pinning centres correlated along the c-axis in PLD-YBCOs film. Supercond Sci Technol 2004; 17:58-64. doi: 10.1088/0953-2048/17/1/010.

5. Zhao Y, Cheng CH, Feng Y, Shibata S, Koshizuka N, Murakami M. The interpretation of improved flux pinning behaviour and second magnetization peaks observed in overdoped Cu-rich Bi2Sr2CaCu208+x single crystals. 2004; 17:S83-S87. doi:10.1088/0953-2048/17/2/068.

6. Misko VR, Savel'ev S, Rakhmanov AL, Nori F. Negative differential resistivity in superconductors with periodic arrays of pinning sites. Phys Rev B. 2007; 75: 024509. doi: 10.1103/PhysRevB.75.024509.

7. Sosnowski J. Superconducting materials: modelling and properties. Warsaw: Electrotechnical Institute Book Publisher; 2008.

8. Sosnowski J. Influence of Radiation Induced Nano-defects on Critical Current of HTc Superconductors. Scientific Journal, Proceedings of the International Conference, Nanomaterials, Applications and Properties; 2012; Ukraina: Sumy State University; 2012.

9. Sosnowski J. Critical current problems in HTc superconductors with nanoscale pinning centers. Proceedings of EUCAS; 2005. Journal of Physics Conf Series 43(1); 2006. p. 659-662. doi: 10.1088/17426596/43/1/161.

10. Koblischka M, Sosnowsk J. Eur Phys JB 2005; 44:270-280.

11. Sosnowski J. Analysis of the pinning interaction in high-temperature superconductors. Mat. Sci. Poland. 2007; 25(4):1081-1085.

12. Mayergoyz ID. Nonlinear diffusion of electromagnetic fields: with applications to eddy currents and superconductivity. San Diego1; Academic Press; 1998. 